\title{
Value of diffusion-weighted imaging combined with conventional magnetic resonance imaging in the diagnosis of thecomas/fibrothecomas and their differential diagnosis with malignant pelvic solid tumors
}

Bing Yin ${ }^{1}$, Wenhua $\mathrm{Li}^{1 *}$, Yanfen Cui ${ }^{1}$, Caiting Chu ${ }^{1}$, Ming Ding ${ }^{1}$, Jian Chen ${ }^{1}$, Ping Zhang ${ }^{2}$ and Xiangru Wu ${ }^{3}$

\begin{abstract}
Background: Our study aims to determine the value of diffusion-weighted imaging (DWI) combined with conventional magnetic resonance imaging (MRI) in the diagnosis of thecomas/fibrothecomas and their differential diagnosis with malignant pelvic solid tumors.

Methods: In total, 36 thecomas/fibrothecomas and 40 malignant pelvic solid tumors were included in our study. All patients underwent $1.5 \mathrm{~T}$ conventional MRI and DWI examinations except one patient with a fibrothecoma in whom DWI examination was not performed. The clinical features and characteristics of conventional MRI and DWI of these two groups were analyzed. Apparent diffusion coefficient (ADC) values were measured and compared between groups. Univariate analysis, multivariate logistic regression analysis, and the receiver operating characteristic curve were used for statistical analysis.

Results: All the thecomas/fibrothecomas showed isointensity on T1 weighted imaging (T1WI) and $77.8 \%$ (28/36) lesions showed hypo- to isointensity on T2 weighted imaging (T2WI). After administration of contrast medium, $94.4 \%$ (34/36) tumors appeared as minor to mild enhancement. On DWI, they showed a diversity of low to very high signal intensity. All malignant pelvic masses manifested as hyperintensity on T2WI and $87.5 \%(35 / 40)$ tumors showed very high signal (grade 3) on DWI. Higher area under the curve (AUC) and specificity could be achieved by using the lowest ADC value than the mean ADC value. Multivariate logistic regression analysis showed that shape, signal intensity on T2WI, capsule, and the lowest ADC value were the important indicators in discriminating thecomas/fibrothecomas from malignant pelvic solid tumors.
\end{abstract}

Conclusions: The combination of DWI and conventional MRI is of great value in the diagnosis of thecomas/ fibrothecomas and their differential diagnosis with malignant pelvic solid tumors.

Keywords: Thecoma, Malignant pelvic solid tumors, Conventional magnetic resonance imaging, Diffusion-weighted imaging, Apparent diffusion coefficient value

\footnotetext{
* Correspondence: wenhualeee@sina.com

${ }^{1}$ Department of Radiology, Xinhua Hospital affiliated to Shanghai Jiao Tong

University School of Medicine, Shanghai 200092, China

Full list of author information is available at the end of the article
} 


\section{Background}

Ovarian thecomas are rare mesothelial tumors with a sex-cord stromal origin, which account for $0.5-1 \%$ of primary ovarian tumors and are the most common solid benign tumors of the ovary [1,2]. Thecomas are composed of theca-like cells with lipid-rich cytoplasm and may have a variable component of fibroblasts (then called fibrothecomas). Thecomas/fibrothecomas are mostly benign tumors and are rarely malignant [3, 4]. They are often asymptomatic and are usually identified incidentally [5]. When symptomatic, patients commonly complain of abdominal pain, abdominal distention, or abdominal masses. They mostly affect menopausal and postmenopausal women but can also occur in young patients with an average age of onset between 55 and 60 years [6]. They may also present with ascites and pleural effusions, known as Meigs syndrome [7, 8].

A correct diagnosis of ovarian thecoma/fibrothecoma is of great value owing to their benign nature. However, given the low incidence and complicated clinical and imaging manifestations of the disease, accurate diagnosis before operating is sometimes difficult. One particular lesion that requires consideration for differential diagnosis is malignant pelvic solid tumors. Though a few cases are mixed cystic-solid and even mainly cystic, most thecomas/fibrothecomas are overwhelmingly solid masses. One of the magnetic resonance imaging (MRI) criteria to diagnose malignant ovarian tumors is a solid mass or a mass with large solid components [2]. Other pathological elements such as edema, degenerative changes, intracellular lipid and varying proportions of theca-like cells and fibroblasts together also contribute to the complexity of their MRI features. In addition, ascites and high levels of CA125 can occur in some patients, which is suggestive of ovarian malignancy in the menopausal women [9]. All the above factors occasionally result in a dilemma in the differential diagnosis of ovarian thecomas/fibrothecomas with malignant pelvic solid tumors. Differential diagnosis of these tumors is of great importance because correct diagnosis of thecomas/fibrothecomas preoperatively can decrease patient anxiety and different diseases will have different patient management strategies. MRI has proven to be a useful method to diagnose thecomas/fibrothecomas. However, reports on MRI features of this disease are extremely limited, especially those including signal characteristics on DWI. To our knowledge, differentiating thecomas/fibrothecomas from malignant pelvic solid masses with apparent diffusion coefficient (ADC) values by a receiver operating characteristic (ROC) curve analysis has not been reported. Therefore, the purposes of our study were to analyze the MRI characteristics of thecomas/fibrothecomas in detail and to differentiate thecomas/fibrothecomas from malignant pelvic solid masses with MRI features including signal characteristics on DWI and ADC values.

\section{Methods \\ Patients}

All ovarian tumors diagnosed by the department of obstetrics and gynecology in our hospital between May 2009 and March 2015 were retrospectively analyzed. In total, 36 thecomas/fibrothecomas (6 thecomas and 30 fibrothecomas) in 34 patients and 40 malignant pelvic solid tumors were included in our study. The 40 malignant pelvic solid tumors include: 22 serous adenocarcinomas, 4 endometroid adenocarcinomas, 3 clear cell adenocarcinomas, 1 undifferentiated carcinoma, 1 dysgerminoma, 2 granular cell tumors, 3 carcinosarcomas, 2 metastatic tumors, and 2 leiomyosarcomas. The disease stage information for the malignant pelvic solid tumors was as follows: stage I, $n=4$; stage II, $n=8$; stage III, $n=21$; and stage IV, $n=7$. The malignant pelvic solid masses were defined as tumors whose solid components accounted for more than $75 \%$ of the entire lesion. All patients were confirmed by surgery and pathology. They all underwent 1.5 T MRI examinations which included conventional precontrast and postcontrast MRI and DWI except one patient with a fibrothecoma in whom DWI was not performed. The study was approved by our institutional ethics committee and written informed consent was obtained from all patients.

\section{MRI protocol}

All patients underwent MRI with a $1.5 \mathrm{~T}$ clinical MR imaging system (Twinspeed, GE Medical Systems, Milwaukee, WI, USA), using a pelvic phased-array coil. The imaging protocol involved axial non-contrast T1 weighted imaging (T1WI) (TR/TE range, 400-600/10$14 \mathrm{~ms}$ ) and axial T2 weighted imaging (T2WI) (TR/TE range, 4000-6000/100-120 ms) performed with a chemical shift-selective fat saturation pulse using the following parameters: slice thickness, $6 \mathrm{~mm}$; gap, $1 \mathrm{~mm}$; field of view (FOV), 32-42 cm; matrix, $256 \times 256$; and number of excitation, 2. Sagittal T1WI and T2WI (TR/TE, 3000-6000/100-110 ms) fast spin-echo imaging without chemical shift-selective fat saturation pulse was also performed, as well as postcontrast enhanced axial and sagittal T1WI with the same parameters, apart from a slice thickness of 5-7 mm. DWI was acquired in the axial plane prior to administration of contrast medium using a single-shot echo-planar imaging sequence (TR/TE effective range, 8000-10,000/70-100; slice thickness/intersection gap, 6/1 mm; FOV, 32 to $42 \mathrm{~cm}$; matrix, $128 \times$ 128; number of excitation, 2). A $b$-value of 0 and $1000 \mathrm{~s} /$ $\mathrm{mm}^{2}$ was also used in three orthogonal ( $\mathrm{Z}, \mathrm{Y}$, and $\mathrm{X}$ ) directions. 


\section{MRI analysis}

MRI data were reviewed independently on an Advantage Windows workstation 4.2 (GE Healthcare, Milwaukee, WI, USA) by two radiologists with 18 and 13 years of experience in gynecological imaging. They both were blind to the pathological results and discrepancies were resolved by consensus. For the two groups of diseases, the following MRI characteristics were recorded: morphology (round/oval, lobulated or irregular); size (the largest diameter measured along the three dimensions); border (clear or not); capsule (present or not); ascites (none, minor, small, large); signal intensity on T1WI and T2WI (hypo-, iso-, hyperintensity, or mixed intensity, compared with the myometrium); degree of enhancement (minor: clearly less than the myometrium; mild: less than the myometrium; marked: similar to the myometrium; severe: more than the myometrium); DWI signal intensity (graded as 1-3; grade 1: low to slightly high signal, obviously lower than the endometrium; grade 2 : high signal, but lower than the endometrium; grade 3: very high, similar to or higher than the endometrium). In addition, for the thecoma/fibrothecomas group, we also evaluated the following features: location (left, right, or both sides); component (solid or mixed cystic/solid); extent of degeneration (none, $<5 \%, 5-30 \%$, and $>30 \%$ of the entire lesion); compared degenerative changes between tumors larger and those smaller than $6 \mathrm{~cm}$; pleural effusion; visibility of ipsilateral ovaries; appearance of peripheral cystic areas; and occurrence of endometrial carcinoma and endometrial hyperplasia (endometrial thickness more than 5 and $16 \mathrm{~mm}$ in postmenopausal and premenopausal women, respectively $[10,11])$.

\section{Data calculation and analysis}

The solid component of each tumor was recognized on T2WI and postcontrast T1WI and was then matched on ADC maps. The ADC values of the solid components of each lesion was measured on diffusion weighted images by one radiologist with the manufacturer's software (FuncTool; GE, Medical Systems, Milwaukee, WI, USA) at an Advantage Windows workstation 4.2 (GE Healthcare). In order to balance variability and error, the largest possible regions of interest (ROIs) ranging from 15 to $500 \mathrm{~mm}^{2}$ were manually placed in the solid portions of the tumors and the degenerative regions were avoided to the best of our ability. No more than three ROIs were drawn in each lesion, and the mean ADC values were calculated and used in our study.

\section{Statistical analysis}

Continuous variables were expressed as means \pm standard deviation (SD). The statistical comparisons for the clinical and image features between groups were conducted by using the univariate analysis (the $\chi^{2}$ test and the Student's $t$ test/Mann-Whitney $U$ test). The ROC curve was also used to compare the ability of the lowest $\mathrm{ADC}$ value and the mean $\mathrm{ADC}$ value in differentiating thecomas/fibrothecomas from malignant pelvic solid masses. The cutoff value was obtained by calculating the maximum Youden index (Youden index = sensitivity + specificity -1 ). The multivariate logistic analysis was performed to show the true prognostic value of ADC value and the other MRI characteristics. Age and lesion size were excluded from the logistic regression analysis because they were not significantly different between the two groups. All analysis was performed with SPSS version 19.0 for Windows (SPSS, Chicago, IL, USA). $P<0.05$ was considered statistically significant.

\section{Results}

\section{Clinical information}

Our research included 36 thecomas/fibrothecomas in 34 patients and 40 malignant pelvic solid tumors. The mean age of patients with thecomas/fibrothecomas was $59.0 \pm$ 13.2 years, and the earliest onset age was 18 years. In the malignant pelvic solid tumor group, the mean patient age was $55.3 \pm 14.5$ years. Most of the thecomas/ fibrothecomas were incidentally identified by health examinations, while the primary complaints included postmenopausal vaginal bleeding and abdominal pain. CA125 tests were performed in 20 of 34 and 31 of 40 patients with thecomas/fibrothecomas and malignant pelvic masses, respectively. Among these, elevated CA125 levels (>35 U/mL) appeared in $25.0 \%(5 / 20)$ and $71.0 \%(22 / 31)$ of patients, respectively.

\section{MRI features and ADC analysis}

In the thecomas/fibrothecomas (Figs. 1, 2, and 3), all lesions showed isointensity on T1WI and $77.8 \%(28 / 36)$ lesions showed hypo- to isointensity on T2WI. In total, $94.4 \%(34 / 36)$ tumors appeared as minor to mild enhancement. On DWI, they showed a diversity of low to very high signal intensity. Solid masses accounted for $86.1 \%(31 / 36)$, while only $13.9 \%(5 / 36)$ tumors were cystic-solid masses (Fig. 4). $58.3 \%$ (21/36) masses showed peripheral cystic areas (Fig. 5). In malignant pelvic tumors (Fig. 6), all manifested as hyperintensity on T2WI and $87.5 \%(35 / 40)$ masses show very high signal (grade 3) on DWI. MRI characteristics of thecomas, fibrothecomas, and malignant pelvic solid masses are summarized in Table 1.

In the univariate analysis, there were significantly statistical differences in shape, signal intensity on T2WI, enhancement degree, pelvic fluid, border, capsule, and the lowest and mean ADC values between groups $(P<0.05)$. Age and lesion size were not significantly different $(P>$ $0.05)$. The lowest and mean ADC value of thecomas/ 


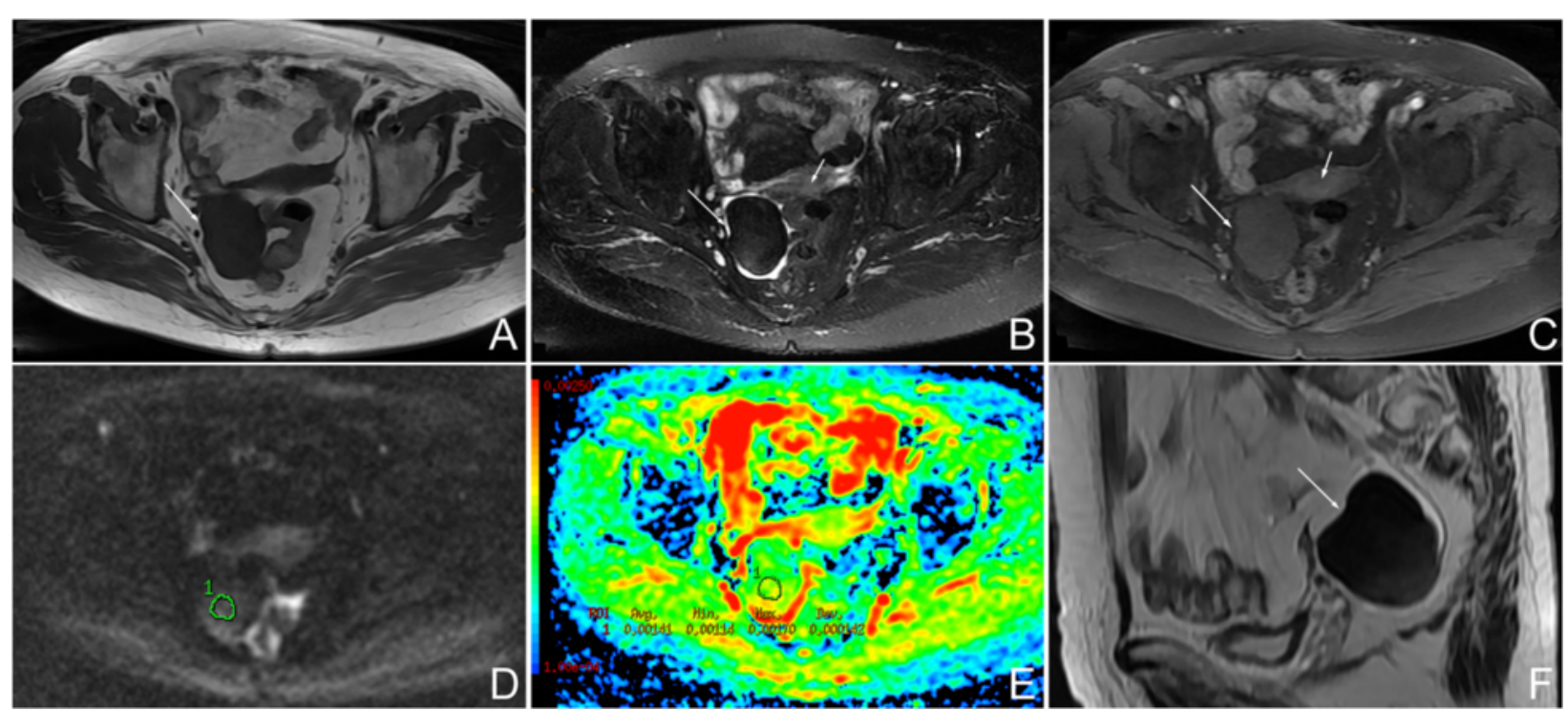

Fig. 1 Female, 77 years, fibrothecoma smaller than $6 \mathrm{~cm}$ on the right ovary. a Axial T1WI showed a solid mass with homogenous isointensity (arrow). b On axial fat saturated T2WI, the mass showed homogenous hypointensity (long arrow) compared with myometrium (short arrow). There was no degenerative change. c On axial contrast-enhanced fat saturated T1WI, the mass (long arrow) revealed minor enhancement, weaker than the myometrium (short arrow). $\mathbf{d}$ On DWI, the mass appeared as a slightly high signal (grade 1). e On ADC map, the mean ADC value was $1.41 \times$ $10^{-3} \mathrm{~mm}^{2} / \mathrm{s}$. f On sagittal T2Wl, the manifestation was similar to Fig. $1 \mathrm{~b}$

fibrothecomas were significantly higher than malignant pelvic solid tumors (lowest ADC: average, $1.16 \pm 0.27 \times$ $10^{-3} \mathrm{~mm}^{2} / \mathrm{s}$ vs $0.81 \pm 0.20 \times 10^{-3} \mathrm{~mm}^{2} / \mathrm{s}$; range, $0.73-$ $1.77 \times 10^{-3} \mathrm{~mm}^{2} / \mathrm{s}$ vs $0.29-1.21 \times 10^{-3} \mathrm{~mm}^{2} / \mathrm{s} ; 95 \%$ confidence interval, $1.07-1.25 \times 10^{-3} \mathrm{~mm}^{2} / \mathrm{s}$ vs $0.74-$ $\left.0.87 \times 10^{-3} \mathrm{~mm}^{2} / \mathrm{s}\right)($ mean ADC: average, $1.25 \pm 0.28 \times 10$ $-3 \mathrm{~mm}^{2} / \mathrm{s}$ vs $0.89 \pm 0.20 \times 10^{-3} \mathrm{~mm}^{2} / \mathrm{s}$; range, $0.73-$ $1.95 \times 10^{-3} \mathrm{~mm}^{2} / \mathrm{s}$ vs $0.42-1.23 \times 10^{-3} \mathrm{~mm}^{2} / \mathrm{s} ; 95 \%$ confidence interval, $1.15-1.34 \times 10^{-3} \mathrm{~mm}^{2} / \mathrm{s}$ vs $0.83-$ $0.96 \times 10^{-3} \mathrm{~mm}^{2} / \mathrm{s}$ ). In the ROC curve analysis, higher
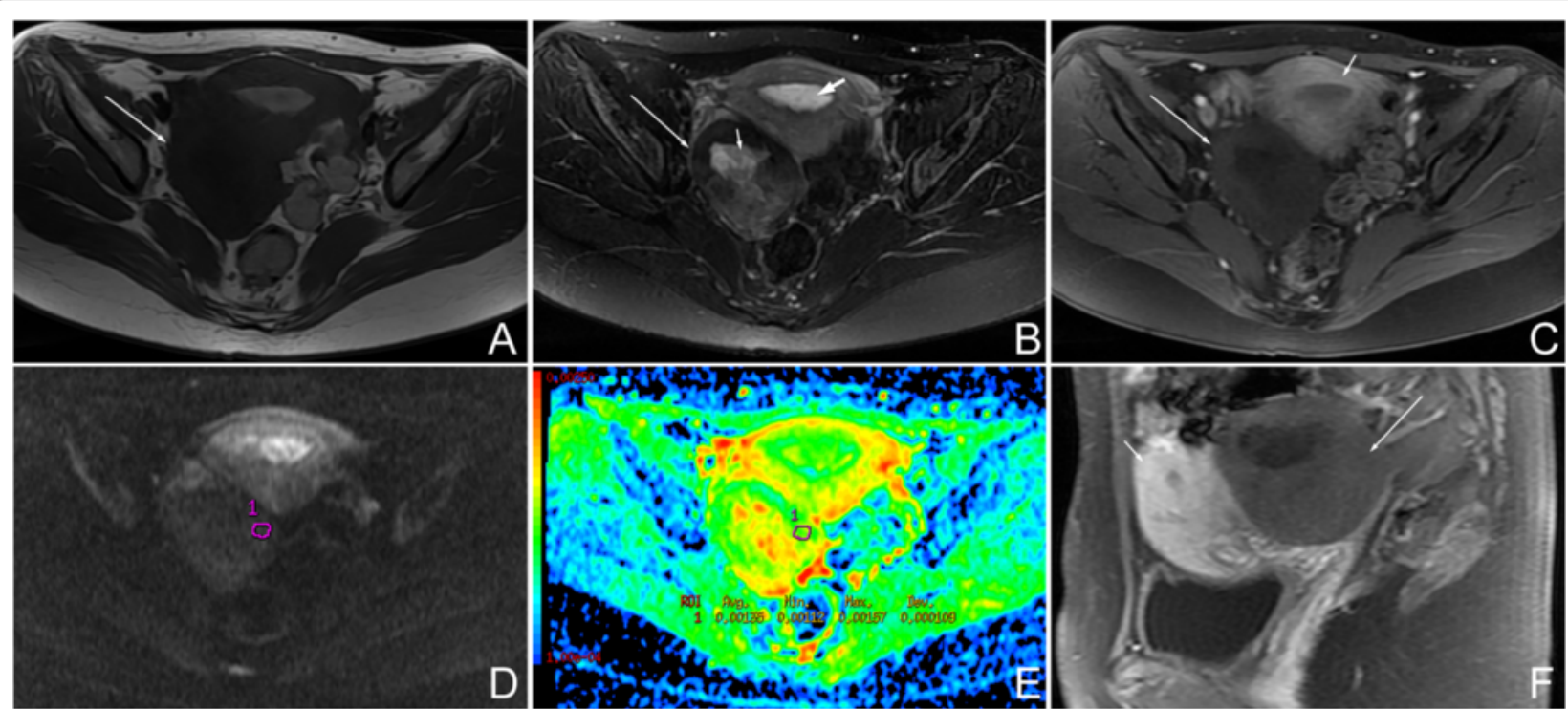

Fig. 2 Female, 27 years, fibrothecoma larger than $6 \mathrm{~cm}$ on the right ovary. a Axial T1WI showed a solid mass with isointensity (arrow). b On axial fat saturated T2WI, the mass (arrow) was hypointensity compared with the myometrium. Hyperintensity in the mass represented degenerative changes (short arrow). This tumor was accompanied with endometrial hyperplasia (bold arrow). c On axial contrast-enhanced fat saturated T1Wl, the mass (long arrow) revealed minor enhancement, weaker than the myometrium (short arrow). $\mathbf{d}$ On DWl, the mass appeared as a slightly high signal (grade 1). e On ADC map, the mean ADC value was $1.35 \times 10^{-3} \mathrm{~mm}^{2} / \mathrm{s}$. f On sagittal contrast-enhanced fat-saturated T1WI, the manifestation was similar to that shown in Fig. $2 \mathrm{C}$ 


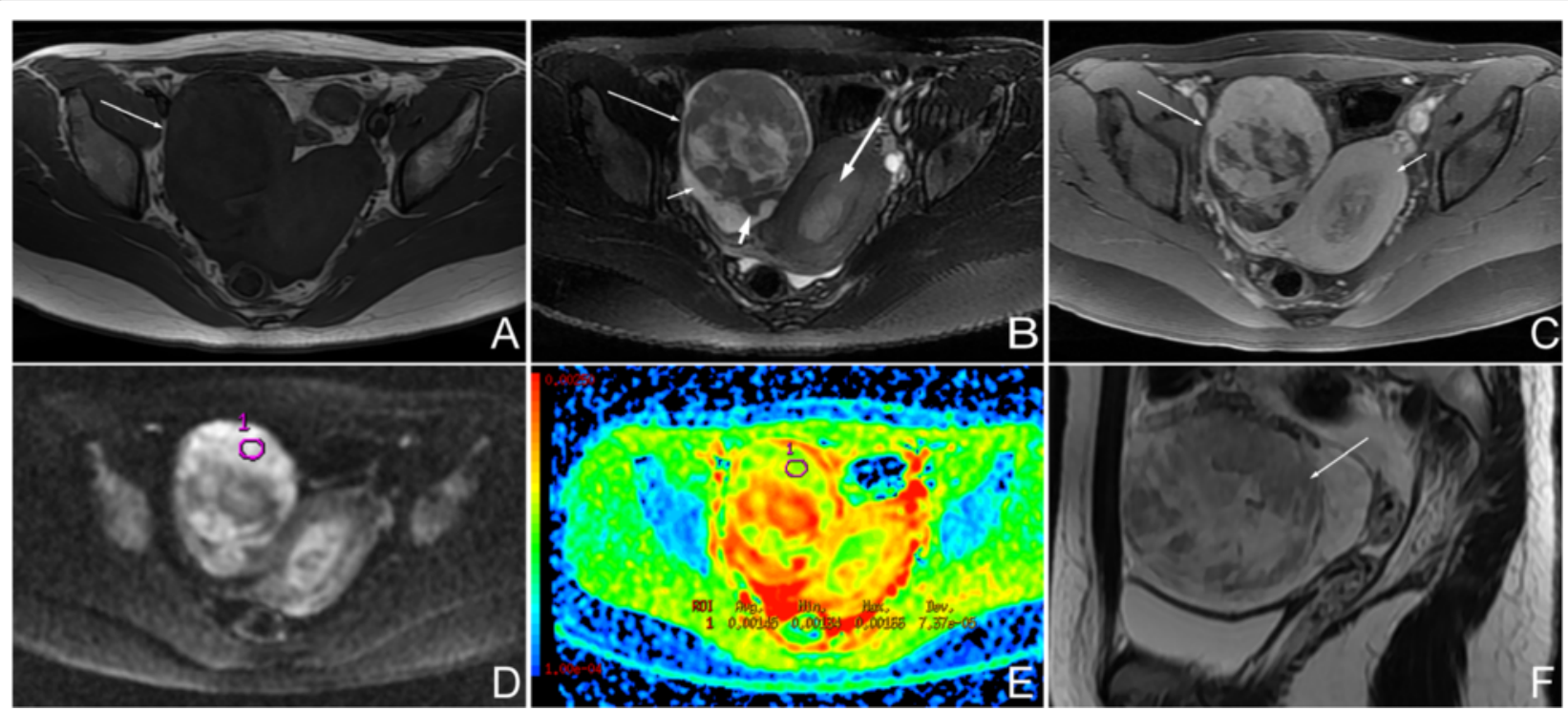

Fig. 3 Female, 18 years, luteinized thecoma on the right ovary. a Axial T1WI showed a relatively large mass with isointensity (arrow). b On axial fat saturated T2Wl, the mass (long arrow) showed heterogeneous isointensity compared with the myometrium. Hyperintensity in the mass represented degenerative changes. Minor-volume of ascites (short arrow) and ipsilateral ovary (short bold arrow) were present. This mass was accompanied with endometrial hyperplasia (long bold arrow). c On axial contrast-enhanced fat-saturated T1WI, the mass revealed marked enhancement (long arrow), similar with the myometrium (short arrow). $\mathbf{d}$ On DWI, the mass showed very high signal (grade 3). e On ADC map, the mean ADC value was $1.45 \times 10$ ${ }^{-3} \mathrm{~mm}^{2} / \mathrm{s}$. $\mathrm{f}$ On sagittal T2WI, the manifestation was similar to Fig. 3b

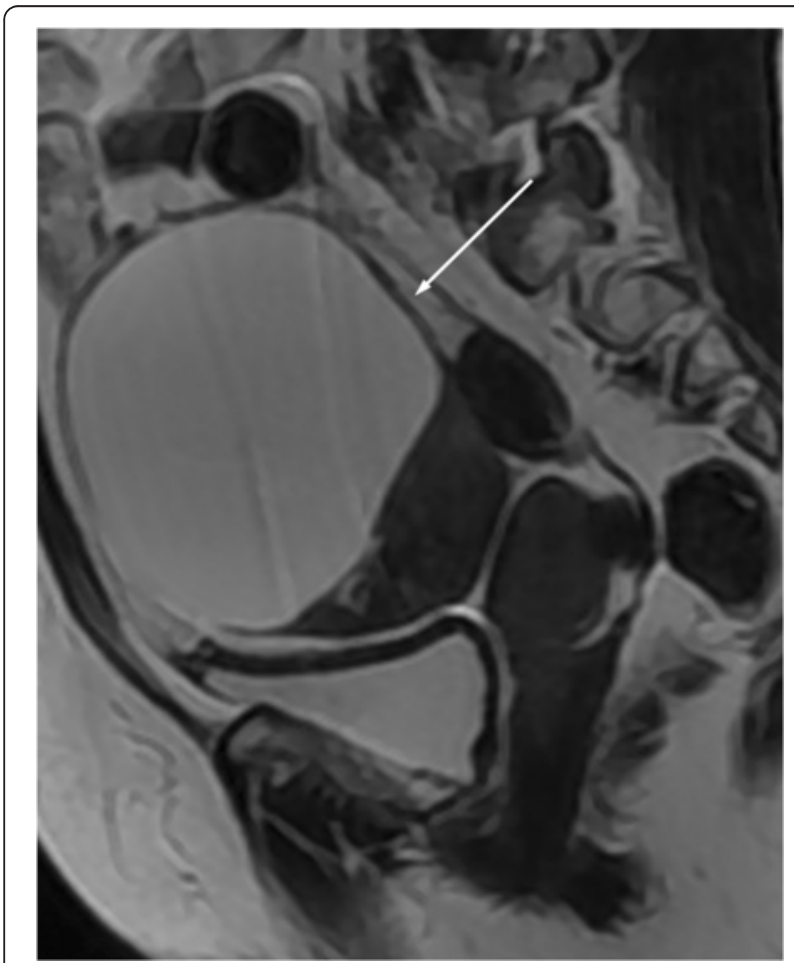

Fig. 4 Female, 59 years, a cystic-solid fibrothecoma on the left side (arrow)

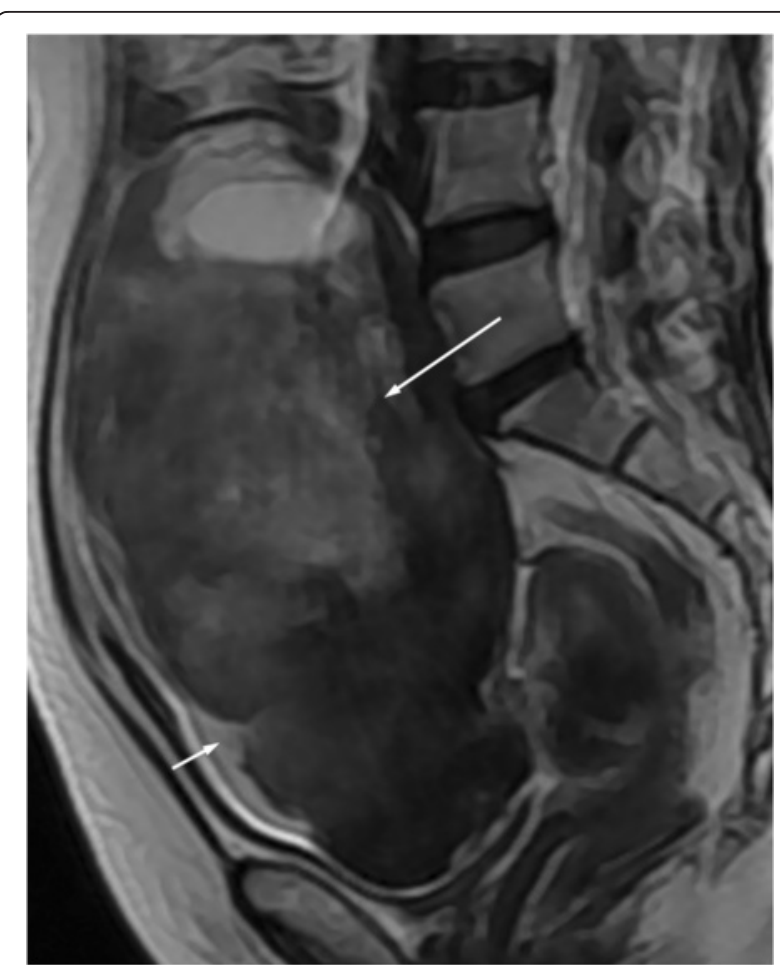

Fig. 5 Female, 63 years, fibrothecoma on the left side (long arrow). Sagittal T2WI showed the peripheral subcapsular cystic areas (short arrow) 


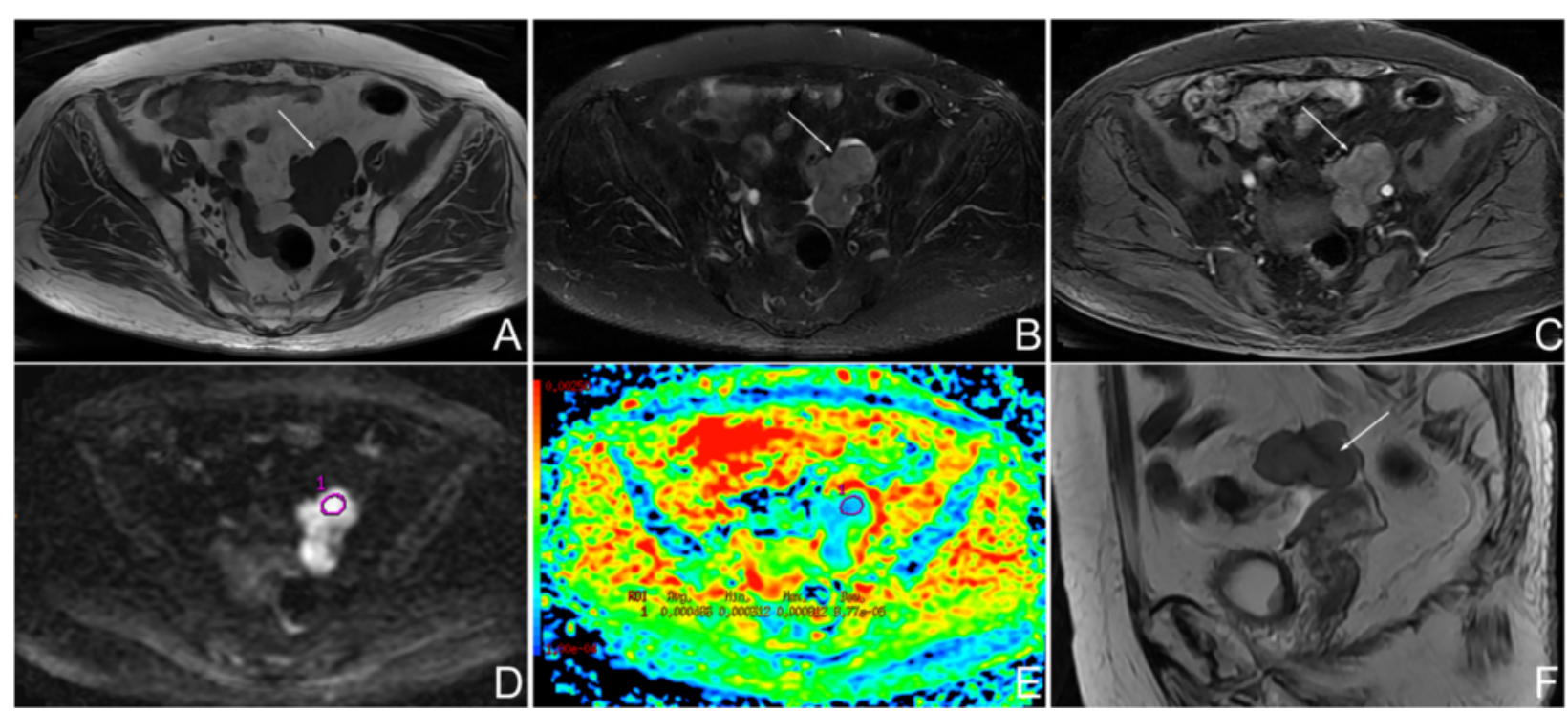

Fig. 6 Female, 85 years, serous adenocarcinoma on the left ovary. a Axial T1WI showed a irregular mass with isointensity (arrow). b On axial fatsaturated T2WI, the mass was hyperintense (arrow) compared with the myometrium (not shown). c On axial contrast-enhanced fat-saturated T1WI, the mass (arrow) revealed less enhancement than myometrium (not show). $\mathbf{d}$ On DWl, the mass appeared as a very high signal (grade 3). e On the ADC map, the mean ADC value was $0.49 \times 10^{-3} \mathrm{~mm}^{2} / \mathrm{s}$. f On sagittal T2WI, the manifestation was similar to Fig. $6 \mathrm{~b}$

AUC and specificity could be achieved by using the lowest $\mathrm{ADC}$ value than the mean $\mathrm{ADC}$ value (lowest $\mathrm{ADC}$ vs mean ADC: cutoff value, $1.02 \times 10^{-3} \mathrm{~mm}^{2} / \mathrm{s}$ vs $0.96 \times$ $10^{-3} \mathrm{~mm}^{2} / \mathrm{s}$; AUC, 0.861 vs $0.842 ; 95 \%$ confidence interval of AUC, 0.781-0.942 vs 0.754-0.930; sensitivity, 71.4 vs $85.7 \%$; specificity, 87.5 vs $67.5 \%$; accuracy: 80.0 vs $76.0 \%$ ) (Fig. 7). The multivariate logistic regression analysis showed that shape, signal intensity on T2WI, capsule, and the lowest ADC value were the significant indicators in discriminating thecomas/fibrothecomas from malignant pelvic solid tumors. The statistical results of the univariate analysis and multivariate logistic regression analysis are summarized in Table 2.

Some other features of thecomas/fibrothecomas are summarized in Table 3. Comparison of degenerative changes between tumors larger and those smaller than $6 \mathrm{~cm}$ is summarized in Table 4.

\section{Discussion}

Thecoma/fibrothecoma is a subtype of sex-cord stromal ovarian tumors with complicated image manifestation that may occasionally result in a misdiagnosis of ovarian malignancy. A correct diagnosis is important because different diagnoses bring about different management strategies. Conventional MRI combined with DWI is confirmed very valuable in the diagnosis of thecomas/ fibrothecomas and in the differential diagnosis between benign and malignant ovarian tumors [12, 13].

Thecomas/fibrothecomas mostly affect menopausal and postmenopausal women, but they can also occur in young patients [6]. They usually grow into relatively large masses, and the adjacent tissues are often compressed [4]. Capsule and border are important aspects in the differential diagnosis between benign and malignant lesions. Results in our study showed that capsule is an independent indicator in differentiating thecomas/ fibrothecomas from malignant pelvic solid tumor. Though border was not significantly different between groups in the multivariate logistic regression, malignancy should still be taken into consideration when an unclear border appeared. In some cases, ascites was present in patients with thecomas/fibrothecomas. In our study, most tumors had accompanying pelvic fluid. Among these patients, $73.5 \%$ only had minor- or small-volume of ascites and only $11.8 \%$ had a large volume. Thecomas/fibrothecomas with ascites and pleural effusions are known as Meigs syndrome. In the present study, only two patients (5.9 \%) had pleural effusions and ascites simultaneously, reflecting the low incidence of this phenomenon; previous literature has shown similar results $[6,14-16]$. In the malignant pelvic masses group, large-volume of ascites occurred in more than half of the patients, a much higher percentage than thecomas/ fibrothecomas. Nevertheless, ascites between groups was not significantly different in the multivariate logistic regression analysis, which indicated that ascites could not be regarded as an independent indicator in their differential diagnosis.

Conventional T1WI and T2WI provide abundant information regarding the pathological features of tumors. In the present study, all thecomas/fibrothecomas showed isointensity on T1WI, which was consistent with a study 
Table 1 Comparison of magnetic resonance imaging characteristics of thecomas, fibrothecomas, and malignant pelvic solid masses

\begin{tabular}{|c|c|c|c|c|}
\hline Image features & Thecoma & Fibrothecoma & Total & Malignant pelvic solid mass \\
\hline \multicolumn{5}{|l|}{ Shape } \\
\hline Round/oval & $83.3 \%(5 / 6)^{a}$ & $70.0 \%(21 / 30)$ & $72.2 \%(26 / 36)$ & $17.5 \%(7 / 40)$ \\
\hline Lobulated & $16.7 \%(1 / 6)$ & $26.7 \%(8 / 30)$ & $25.0 \%(9 / 36)$ & $7.5 \%(3 / 40)$ \\
\hline Irregular & 0 & $3.3 \%(1 / 30)$ & $2.8 \%(1 / 36)$ & $75.0 \%(30 / 40)$ \\
\hline \multicolumn{5}{|l|}{ Signal intensity } \\
\hline \multicolumn{5}{|l|}{ T1WI } \\
\hline Iso- & $100 \%(6 / 6)$ & $100 \%(30 / 30)$ & $100 \%(36 / 36)$ & $100 \%(40 / 40)$ \\
\hline \multicolumn{5}{|l|}{$\mathrm{T} 2 \mathrm{Wl}$} \\
\hline Hypo- & $16.7 \%(1 / 6)$ & $60.0 \%(18 / 30)$ & $52.8 \%(19 / 36)$ & 0 \\
\hline Iso- & $50.0 \%(3 / 6)$ & $20.0 \%(6 / 30)$ & $25.0 \%(9 / 36)$ & 0 \\
\hline Hyper- & $33.3 \%(2 / 6)$ & 0 & $5.6 \%(2 / 36)$ & $100 \%(40 / 40)$ \\
\hline Mixed & 0 & $20.0 \%(6 / 30)$ & $16.7 \%(6 / 36)$ & 0 \\
\hline \multicolumn{5}{|c|}{ Enhancement degree } \\
\hline Minor & 0 & $70.0 \%(21 / 30)$ & $58.3 \%(21 / 36)$ & $10.0 \%(4 / 40)$ \\
\hline Mild & $66.7 \%(4 / 6)$ & $30.0 \%(9 / 30)$ & $36.1 \%(13 / 36)$ & $50.0 \%(20 / 40)$ \\
\hline Marked & $33.3 \%(2 / 6)$ & 0 & $5.6 \%(2 / 36)$ & $35.0 \%(14 / 40)$ \\
\hline Severe & 0 & 0 & 0 & $5.0 \%(2 / 40)$ \\
\hline \multicolumn{5}{|l|}{ DWI signal } \\
\hline Grade 1 & 0 & $65.5 \%(19 / 29)$ & $54.3 \%(19 / 35)$ & $2.5 \%(1 / 40)$ \\
\hline Grade 2 & $33.3 \%(2 / 6)$ & $27.6 \%(8 / 29)$ & $28.6 \%(10 / 35)$ & $10.0 \%(4 / 40)$ \\
\hline Grade 3 & $66.7 \%(4 / 6)$ & $6.9 \%(2 / 29)$ & $17.1 \%(6 / 35)$ & $87.5 \%(35 / 40)$ \\
\hline \multicolumn{5}{|l|}{ Pelvic fluid } \\
\hline None & 0 & $17.9 \%(5 / 28)$ & $14.7 \%(5 / 34)$ & $12.5 \%(5 / 40)$ \\
\hline Present & $100 \%(6 / 6)$ & $82.1 \%(23 / 28)$ & 85.3 \%(29/34) & $87.5 \%(35 / 40)$ \\
\hline Minor & $50.0 \%(3 / 6)$ & $46.4 \%(13 / 28)$ & $47.1 \%(16 / 34)$ & $15.0 \%(6 / 40)$ \\
\hline Small & $33.3 \%(2 / 6)$ & $25.0 \%(7 / 28)$ & $26.5 \%(9 / 34)$ & $15.0 \%(6 / 40)$ \\
\hline Large & $16.7 \%(1 / 6)$ & $10.7 \%(3 / 28)$ & $11.8 \%(4 / 34)$ & $57.5 \%(23 / 40)$ \\
\hline \multicolumn{5}{|l|}{ Border } \\
\hline Clear & $100 \%(6 / 6)$ & $100 \%(30 / 30)$ & $100 \%(36 / 36)$ & $27.5 \%(11 / 40)$ \\
\hline Not clear & 0 & 0 & 0 & $72.5 \%(29 / 40)$ \\
\hline \multicolumn{5}{|l|}{ Capsule } \\
\hline Present & $100 \%(6 / 6)$ & $96.7 \%(29 / 30)$ & $97.2 \%(35 / 36)$ & $52.5 \%(21 / 40)$ \\
\hline Absent & 0 & $3.3 \%(1 / 30)$ & $2.8 \%(1 / 36)$ & $47.5 \%(19 / 40)$ \\
\hline
\end{tabular}

T1WI T1 weighted imaging, T2WI T2 weighted imaging, DWI diffusion-weighted imaging, hypo hypointensity, iso isointensity, hyper hyperintensity

a Percentage

performed by Zhang et al. [17]. On T2WI, hypo-, iso-, hyper-, and mixed intensity occurred in 52.8, 25.0, 5.6, and $16.7 \%$ of tumors, respectively. In a study conducted by Chung et al. [12], 55.6 and $44.4 \%$ thecomas/fibrothecomas showed low and high signal intensity on T2WI, respectively, which was comparable with our study. In our opinion, the hypointensity on T2WI were associated with the component of abundant fibroblasts and the relatively hyperintensity had some relationship with the lipid containing theca-like cells or stromal edema in the tumors. The variable proportion of theca-like cells and fibroblasts contributed to the mixture of manifestations on T2WI. In the malignant pelvic solid masses group, all patients showed isointensity on T1WI and hyperintensity on T2WI. All these results indicate that isointensity on T1WI and hypo- to isointensity on T2WI is the characteristic manifestation of thecomas/fibrothecomas and hyperintensity on T2WI was a significant indicator of pelvic malignant solid tumors $(P=0.006, \mathrm{OR}=117.274)$. 


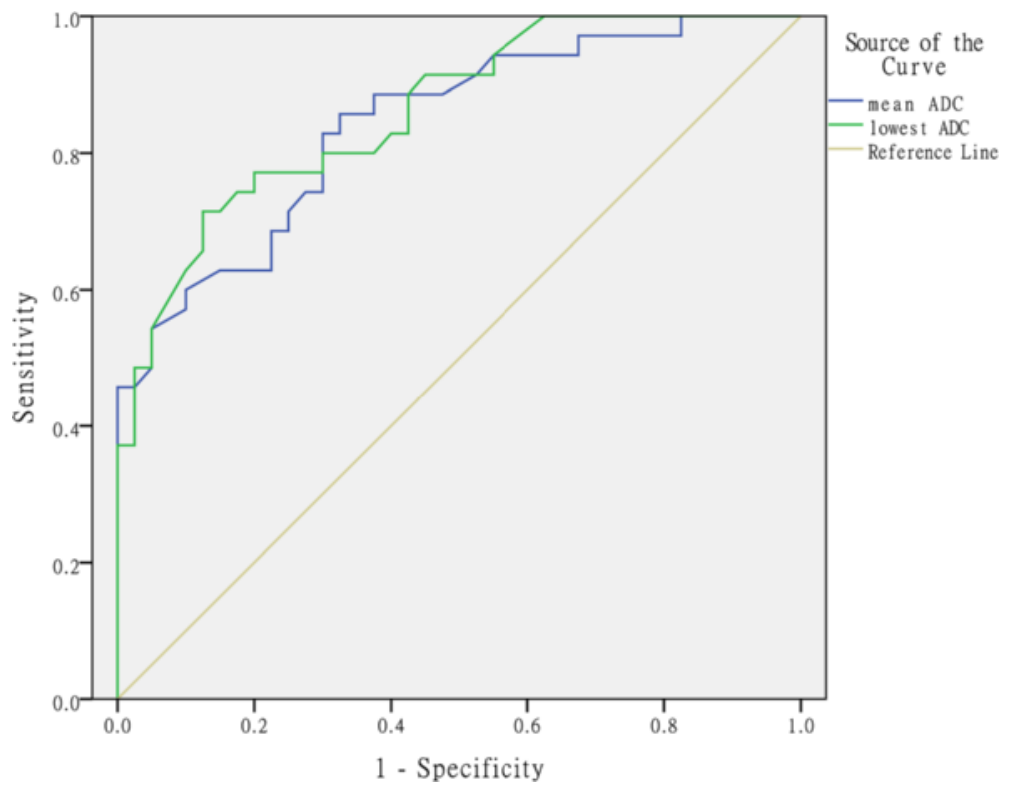

Fig. 7 Comparative diagnostic value of different ADC values for differentiating thecomas/fibrothecomas from malignant pelvic solid tumors

Previous studies $[3,4,7,14,17]$ confirmed that thecomas/fibrothecomas in most cases showed minor to mild enhancement due to poor blood supply. In our study, $58.3 \%(21 / 36)$ and $36.1 \%(13 / 36)$ of masses showed minor and mild enhancement, respectively, which was comparable to previous studies. Only $5.6 \%(2 / 36)$ of tumors showed marked enhancement in the present study and both of them were thecomas, which indicates that fibrothecomas with more theca-like cell components seemed to showed higher enhancement and it was more likely to be a thecoma than fibrothecoma when a lesion appears with marked enhancement [7]. Conversely, in malignant pelvic masses, the enhancement degree was variable owing to the diversity of tumor constitution. The multivariate logistic regression analysis showed that enhancement degree was not a significant indicator in the differential diagnosis between groups, which may be caused by the tumor constitution diversity of the malignant pelvic solid tumors.

Despite the characteristics observed with conventional MRI approaches, it is still difficult to differentiate these two groups of diseases. DWI is a functional imaging method that reflects water molecule motion and is recently used to improve the diagnostic accuracy of MRI in pelvic masses $[13,18]$. The movement of water molecules in body tissues is impeded by interactions with cell membranes, intracellular organelles, matrix fibers, and hydrophobic macromolecules. Moreover, ADC value is a quantitative expression to reflect water diffusion in tissues. Generally, when high signal on DWI or low ADC values are detected, a malignancy or the presence of hypercellularity should be taken into consideration [13,
19-21]. DWI signal intensity and ADC values of benign and malignant tumors should be different owing to their different pathological bases, thus aiding in discriminating thecomas/fibrothecomas from other malignant pelvic solid tumors.

In the present study, grade 1 to 3 signal on DW-MRI occurred in $54.3 \%$ (19/35), $28.6 \%(10 / 35)$, and $17.1 \%$ (6/35) of thecomas/fibrothecomas, respectively. Grade 1 and grade 3 signals differed significantly between thecomas/fibrothecomas and malignant pelvic masses (54.3 vs $2.5 \%, 17.1$ vs $87.5 \%$, respectively), which were of great importance in their differential diagnosis. In our cohort, DWI signal intensity of thecomas/fibrothecomas varied from low to very high, which may be associated with the variable component of fibroblasts and thecalike cells. Notably, all six thecomas showed grade 2 and 3 signal intensity on DWI, and grade 1 signal intensity was not present. In a study performed by Chung et al. [12], low and high signal on DWI in fibromas accounted for 93.3 and $6.7 \%$ while in thecomas/fibrothecomas, they accounted for 28.6 and $71.4 \%$, respectively. Their study also showed that both thecomas included in their study showed high intensity on DWI, which is in agreement with our results. Together, our studies indicate that thecomas/fibrothecomas with more theca-like cell content and less fibroblast content tend to have relatively high signal intensity on DWI and vice versa. Therefore, thecomas are more likely to have high signals, fibromas tend to show low signal, and fibrothecomas can show a mixture of low to high signals on DWI.

As mentioned previously, ADC value is a quantitative expression of the diffusion characteristics of tissues and 
Table 2 The statistical results of the univariate analysis and multivariate logistic regression analysis

\begin{tabular}{|c|c|c|c|c|c|c|c|c|c|c|c|}
\hline \multirow[t]{2}{*}{ Indicators } & \multirow{2}{*}{$\begin{array}{l}\text { Thecoma/ } \\
\text { fibrothecoma }\end{array}$} & \multirow{2}{*}{$\begin{array}{l}\text { Malignant } \\
\text { pelvic } \\
\text { solid mass }\end{array}$} & \multirow{2}{*}{$\begin{array}{l}\text { Univariate } \\
\text { analysis (P) }\end{array}$} & \multicolumn{8}{|c|}{ Multivariate logistic regression analysis } \\
\hline & & & & $\mathrm{B}$ & S.E. & Wals & $\mathrm{df}$ & $P$ & OR & ${\text { Lower } \text { limit }^{\mathrm{a}}}$ & Upper limit ${ }^{a}$ \\
\hline Age (years) & $58.5 \pm 12.7$ & $55.3 \pm 14.5$ & 0.302 & & & & & - & & & \\
\hline Size $(\mathrm{mm})$ & $70.3 \pm 41.8$ & $78.7 \pm 39.5$ & 0.376 & & & & & - & & & \\
\hline Shape & & & $<0.001$ & & & 7.977 & 2 & 0.019 & & & \\
\hline Round/oval & 25 & 7 & & & & & & Reference & & & \\
\hline Lobulated & 9 & 3 & & -0.676 & 1.582 & 0.182 & 1 & 0.669 & 0.509 & 0.023 & 11.299 \\
\hline Irregular & 1 & 30 & & 7.652 & 2.710 & 7.974 & 1 & 0.005 & 2105.780 & 10.390 & 426777.060 \\
\hline $\mathrm{T} 2 \mathrm{Wl}$ & & & $<0.001$ & 4.765 & 1.718 & 7.695 & 1 & 0.006 & 117.274 & 4.048 & 3397.444 \\
\hline Hypo- & 18 & 0 & & & & & & & & & \\
\hline Iso- & 9 & 0 & & & & & & & & & \\
\hline Hyper- & 2 & 40 & & & & & & & & & \\
\hline Mixed & 6 & 0 & & & & & & & & & \\
\hline Enhancement degree & & & $<0.001$ & & & & & 0.256 & & & \\
\hline Minor & 20 & 4 & & & & & & Reference & & & \\
\hline Mild & 13 & 20 & & & & & & 0.149 & & & \\
\hline Marked & 2 & 14 & & & & & & 0.062 & & & \\
\hline Severe & 0 & 2 & & & & & & 1.000 & & & \\
\hline Pelvic fluid & & & 0.001 & & & & & 0.436 & & & \\
\hline None & 4 & 5 & & & & & & Reference & & & \\
\hline Minor & 16 & 6 & & & & & & 0.241 & & & \\
\hline Small & 9 & 6 & & & & & & 0.981 & & & \\
\hline Large & 4 & 23 & & & & & & 0.490 & & & \\
\hline Border & & & $<0.001$ & 21.936 & 2973.934 & 0.000 & 1 & 0.994 & 3.364E9 & 0.000 & \\
\hline Clear & 35 & 11 & & & & & & & & & \\
\hline Not clear & 0 & 29 & & & & & & & & & \\
\hline Capsule & & & $<0.001$ & 6.105 & 2.972 & 4.221 & 1 & 0.040 & 448.142 & 1.324 & 151649.370 \\
\hline Present & 34 & 21 & & & & & & & & & \\
\hline Absent & 1 & 19 & & & & & & & & & \\
\hline Lowest ADC & & & $<0.001$ & 8.775 & 3.208 & 7.483 & 1 & 0.006 & 6467.870 & 12.032 & 3476890.705 \\
\hline$>1.02^{b}$ & 25 & 5 & & & & & & & & & \\
\hline$<1.02^{b}$ & 10 & 35 & & & & & & & & & \\
\hline
\end{tabular}

One case lacking the data of ADC values was not used in the statistical analysis

$B$ Value partial regression coefficient, S.E. partial regression coefficient standard errors, $O R$ odds ratio

${ }^{a} 95 \% \mathrm{Cl}$ (confidence interval) of $\mathrm{OR}$

${ }^{\mathrm{b}} \times 10^{-3} \mathrm{~mm}^{2} / \mathrm{s}$

provides the optimal application of imaging method for the quantitative evaluation of different lesions in vivo. $\mathrm{ADC}$ value has been proved to be a useful tool in the differential diagnosis between benign and malignant lesions [22-24]. In our study, the lowest and mean ADC values of thecomas/fibrothecomas were significantly higher than that of malignant pelvic solid masses $(P<$ 0.001). Higher AUC and specificity could be achieved by using the lowest $A D C$ value than the mean $A D C$ value, which indicated the former performed better in this aspect. Multivariate logistic regression analysis showed that the lowest ADC value was a significant indicator in the differential diagnosis between groups $(P=0.006$, OR $=6467.870)$. These results implied that ADC value, especially the lowest ADC value, was very valuable in distinguishing thecomas/fibrothecomas from malignant pelvic solid tumors. However, in a study conducted by Zhang et al. [17], there was no significant difference in ADC values between thecomas/fibrothecomas and other solid ovarian masses, even between benign and malignant masses. This discrepancy may be associated with different $b$ values selected $\left(b=700 \mathrm{~mm}^{-2} / \mathrm{s}\right.$ in their study) and 
Table 3 Some other features of thecomas/fibrothecomas

\begin{tabular}{llll}
\hline Image features & Thecoma & Fibrothcoma & Total \\
\hline $\begin{array}{llll}\text { Component } \\
\quad \text { Solid }\end{array}$ & $100 \%(6 / 6)^{\mathrm{a}}$ & $83.3 \%(25 / 30)$ & $86.1 \%(31 / 36)$ \\
$\quad$ Cystic-solid & 0 & $16.7 \%(5 / 30)$ & $13.9 \%(5 / 36)$ \\
Degenerative changes & & & \\
$\quad$ None & $50.0 \%(3 / 6)$ & $33.3 \%(10 / 30)$ & $36.1 \%(13 / 36)$ \\
$\quad$ Present & $50.0 \%(3 / 6)$ & $66.7 \%(20 / 30)$ & $63.9 \%(23 / 36)$ \\
$\quad<5 \%$ & 0 & $36.7 \%(11 / 30)$ & $30.6 \%(11 / 36)$ \\
$\quad 5-30 \%$ & $33.3 \%(2 / 6)$ & $26.7 \%(8 / 30)$ & $27.8 \%(10 / 36)$ \\
$\quad>30 \%$ & $16.7 \%(1 / 6)$ & $3.3 \%(1 / 30)$ & $5.6 \%(2 / 36)$ \\
Peripheral cystic areas & $83.3 \%(5 / 6)$ & $53.3 \%(16 / 30)$ & $58.3 \%(21 / 36)$ \\
Ipsilateral ovary & $33.3 \%(2 / 6)$ & $33.3 \%(10 / 30)$ & $33.3 \%(12 / 36)$ \\
Endometrial hyperplasia & $33.3 \%(2 / 6)$ & $3.6 \%(1 / 28)$ & $8.8 \%(3 / 34)$ \\
\hline aPercentage & & &
\end{tabular}

different tumor constitutions of pelvic solid masses selected in our two cohorts (five benign tumors were included in their cohort). Besides, manually placing the ROI in our two studies had the potential risk of measurement error. In the present study, the relatively low ADC values of thecomas/fibrothecomas likely have something to do with T2 black out effect, densely arranged spindle cells and thecal cells, and network of collagen fibers within the extracellular matrix. However, owing to their benign nature, the ADC value of thecomas/fibrothecomas was still apparently higher than malignant pelvic solid masses. When the lowest $\mathrm{ADC}$ value of $1.02 \times 10^{-3} \mathrm{~mm}^{2} / \mathrm{s}$ was used as the cutoff value, sensitivity, specificity, and the highest accuracy were 71.4 , 87.5 , and $80.0 \%$, respectively. To the best of our knowledge, no studies have determined and compared the validity of different $\mathrm{ADC}$ values in differentiating thecomas/fibrothecomas from malignant pelvic solid masses by the ROC curve analysis. All these results confirmed the great value of ADC measurements in the differential diagnosis between these two groups of diseases.

Some other characteristics of thecomas/fibrothecomas include (1) Component: thecomas/fibrothecomas are mostly solid masses and predominantly cystic or mixed cystic-solid ones are rare. In the present study, cysticsolid tumors accounted for only $13.9 \%$ (5/36) of cases.

Table 4 Comparison of degenerative changes between tumors larger and those smaller than $6 \mathrm{~cm}$

\begin{tabular}{lll}
\hline & $<6 \mathrm{~cm}$ & $>6 \mathrm{~cm}$ \\
\hline None & $66.7 \%(12 / 18)$ & $5.6 \%(1 / 18)$ \\
Present & $33.3 \%(6 / 18)$ & $94.4 \%(17 / 18)$ \\
$<5 \%$ & $22.2 \%(4 / 18)$ & $38.9 \%(7 / 18)$ \\
$5-30 \%$ & $11.1 \%(2 / 18)$ & $44.4 \%(8 / 18)$ \\
$>30 \%$ & 0 & $11.1 \%(2 / 18)$ \\
\hline
\end{tabular}

(2) Degenerative changes: degenerative changes can occur due to insufficient blood supply as tumors grow. In the present study, degenerative changes presented in $63.9 \%(23 / 36)$ of patients, among which tiny degenerative changes $(<5 \%)$ accounted for $30.6 \%(11 / 36)$. It has been previously reported that thecomas/fibrothecomas larger than $6 \mathrm{~cm}$ more often show degenerative changes $[3,7$, 14]. Our study was in agreement with these reports. In our research, degenerative changes occurred in $94.4 \%$ $(17 / 18)$ lesions larger than $6 \mathrm{~cm}$, while in lesions $<6 \mathrm{~cm}$, the incidence rate was $33.3 \%$ (6/18). (3) Peripheral subcapsular cystic areas: in a study conducted by Shinagare et al. [7], peripheral subcapsular cystic areas, which represented edematous change on pathologic examination, occurred in $49 \%$ of tumors. In the present study, $58.3 \%$ of thecomas/fibrothecomas showed this feature. (4) Ipsilateral ovary: in our study, the ipsilateral ovary was seen in $33.3 \%(12 / 36)$ patients, which was similar to a study by Oh et al. (46 \%) [25]. (5) Endometrial hyperplasia and endometrial carcinoma: thecomas/fibrothecomas are frequently estrogenic and are occasionally accompanied with associated manifestations such as endometrial hyperplasia and endometrial carcinoma [4, 7]. In a report by Li et al. [4], endometrial hyperplasia and endometrial carcinoma occurred in 10.5 and $5.2 \%$ of patients with thecomas/ fibrothecomas. In our study, $8.8 \%(3 / 34)$ of patients had endometrial hyperplasia and no patient had endometrial carcinoma. (6) Luteinized thecomas: luteinized thecomas are uncommon and include any thecomas that containing luteinized cells. They more often occur in younger patients than typical thecomas, and $30 \%$ of patients with luteinized thecomas present under the age of 30 years [26, 27]. In our study, luteinized thecoma occurred in one female 18 years of age.

There were several limitations to our study. First, because of the retrospective nature of our study, biases may inevitably occur and some prospective studies should be performed in future. Second, the semiquantitative evaluation of some characteristics like ascites and degenerative changes of the tumors in the present study was not accurate enough owing to the lack of reliable measurement methods. Third, time-signal intensity curve were not obtained after administration of contrast medium in our study and perfusion weighted image (PWI) should be added in the subsequent studies. In addition, though the sample size in our study is the largest among the literature focusing on MRI characteristics of thecomas/fibrothecomas, further work with a larger sample size is still needed to provide reliable reference for gynecologists. Nevertheless, our study also has some positive results. First, to the best of our knowledge, our study is one of the very few reporting DWI characteristics of thecomas/fibrothecomas, and both of the previous studies had very small sample sizes $(n=18$ and 
9, respectively) [12, 17]. Second, our study is the first to differentiate thecomas/fibrothecomas from malignant pelvic solid masses using different ADC values by the ROC curve analysis.

\section{Conclusions}

In summary, thecomas/fibrothecomas are rare benign ovarian tumors and occasionally are misdiagnosed as pelvic malignancies. Most thecomas/fibrothecomas show isointensity on T1WI and hypo/isointensity on T2WI with minor to mild enhancement. They are usually round or oval with a clear margin, and degenerative changes occur more often in larger lesions. Ascites and elevated CA125 levels can occur in some cases as well. Conversely, most malignant pelvic masses are hyperintensity on T2WI with irregular morphology and unclear border. Signal intensity on DWI is useful in their differential diagnosis. Shape, signal intensity on T2WI, capsule, and the lowest ADC value were significant indicators in the differential diagnosis between groups. The combination of DWI and conventional MRI is of great value in the diagnosis of thecomas/ fibrothecomas and their differential diagnosis with malignant pelvic solid tumors.

\section{Competing interests}

This work was supported by the Science and Technology Commission of Shanghai Municipality, NO.15ZR1427500. The authors declare that they have no conflict of interest.

\section{Authors' contributions}

WL designed the study, analyzed the data, and revised the manuscript. BY designed the study, collected the data, and drafted the manuscript. YC analyzed the data and revised the manuscript. CC, MD, and JC collected and analyzed the data. PZ and XW contributed as a gynecologist and pathologist and helped in writing the manuscript. All authors read and approved the final manuscript.

\section{Acknowledgements}

We thank Qiufeng Yin, Ming Liu, and Jiashu Wu for their technical support.

\begin{abstract}
Author details
${ }^{1}$ Department of Radiology, Xinhua Hospital affiliated to Shanghai Jiao Tong University School of Medicine, Shanghai 200092, China. ${ }^{2}$ Department of Obstetrics and Gynecology, Xinhua Hospital affiliated to Shanghai Jiao Tong University School of Medicine, Shanghai 200092, China. ${ }^{3}$ Department of Pathology, Xinhua Hospital affiliated to Shanghai Jiao Tong University School of Medicine, Shanghai 200092, China.
\end{abstract}

Received: 28 August 2015 Accepted: 30 December 2015

Published online: 08 January 2016

\section{References}

1. Okada I, Nakagawa S, Takemura Y, Takenobu T, Matsuoka T, Mandai H, et al. Ovarian thecoma associated in the first trimester of pregnancy. J Obstet Gynaecol Res. 2004;30:368-71.

2. Imaoka I, Wada A, Kaji Y, Hayashi T, Hayashi M, Matsuo M, et al. Developing an MR imaging strategy for diagnosis of ovarian masses. Radiographics. 2006:26:1431-48.

3. Troiano RN, Lazzarini KM, Scoutt LM, Lange RC, Flynn SD, McCarthy S. Fibroma and fibrothecoma of the ovary: MR imaging findings. Radiology. 1997;204:795-8.

4. Li X, Zhang W, Zhu G, Sun C, Liu Q, Shen Y. Imaging features and pathologic characteristics of ovarian thecom. J Comput Assit Tomogr. 2012;36:46-53.
5. Sivanesaratnam V, Dutta R, Jayalakshmi P. Ovarian fibroma: clinical and histopathological characteristics. Int J Gynaecol Obstet. 1990;33:243-7.

6. Yen P, Khong K, Lamba R, Corwin MT, Gerscovich EO. Ovarian fibromas and fibrothecomas: sonographic correlation with computed tomography and magnetic resonance imaging: a 5-year singleinstitution experience. J Ultrasound Med. 2013;32:13-8.

7. Shinagare $A B$, Meylaerts $L$, Laury AR, Mortele KJ. MRI features of ovarian fibroma and fibrothecoma with histopathologic correlation. AJR Am J Roentgenol. 2012;198:296-303.

8. Tanaka YO, Tsunoda H, Kitagawa Y, Ueno T, Yoshikawa H, Saida Y. Functioning ovarian tumors: direct and indirect findings at MR imaging. Radiographics. 2004;24 Suppl 1:147-66.

9. Yazdani S, Alijanpoor A, Sharbatdaran M, Bouzari Z, Abedisamaoosh M, Lakaieandi F, et al. Meigs' syndrome with elevated serum CA125 in a case of ovarian fibroma/thecoma. Caspian J Intern Med. 2014:5:43-5.

10. Ferrazzi E, Torri V, Trio D, Zannoni E, Filiberto S, Dordoni D. Sonographic endometrial thickness: a useful test to predict atrophy in patients with postmenopausal bleeding? An Italian multicenter study. Ultrasound Obstet Gynecol. 1996;7:315-21.

11. Fleischer AC. Sonographic assessment of endometrial disorders. Semin Ultrasound CT MR. 1999;20:259-66.

12. Chung BM, Park SB, Lee JB, Park HJ, Kim YS, Oh YJ. Magnetic resonance imaging features of ovarian fibroma, fibrothecoma, and thecoma. Abdom Imaging. 2015:40:1263-72.

13. Li W, Chu C, Cui Y, Zhang P, Zhu M. Diffusion-weighted MRl: a useful technique to discriminate benign versus malignant ovarian surface epithelial tumors with solid and cystic components. Abdom Imaging. 2012;37:897-903.

14. Zhang Z, Wu Y, Gao J. CT diagnosis in the thecoma-fibroma group of the ovarian stromal tumors. Cell Biochem Biophys. 2015;71:937-43.

15. Agaba El, Ekwempu CC, Ugoya SO, Echejoh GO. Meigs' syndrome presenting as haemorrhagic pleural effusion. West Afr J Med. 2007;26:253-5.

16. Macciò A, Madeddu C, Kotsonis P, Pietrangeli M, Paoletti AM. Large twisted ovarian fibroma associated with Meigs' syndrome, abdominal pain and severe anemia treated by laparoscopic surgery. BMC Surg. 2014;14:38.

17. Zhang $H$, Zhang GF, Wang TP, Zhang H. Value of $3.0 \mathrm{~T}$ diffusion-weighted imaging in discriminating thecoma and fibrothecoma from other adnexal solid masses. J Ovarian Res. 2013:6:58.

18. Punwani S. Diffusion weighted imaging of female pelvic cancers: concepts and clinical applications. Eur J Radiol. 2011;78:21-9.

19. Dhanda S, Thakur M, Kerkar R, Jagmohan P. Diffusion-weighted imaging of gynecologic tumors: diagnostic pearls and potential pitfalls. Radiographics. 2014;35:1393-416.

20. Zhang P, Cui Y, Li W, Ren G, Chu C, Wu X. Diagnostic accuracy of diffusionweighted imaging with conventional MR imaging for differentiating complex solid and cystic ovarian tumors at 1.5T. World J Surg Oncol. 2012;10:237.

21. Zhang H, Zhang GF, He ZY, Li ZY, Zhu M, Zhang GX. Evaluation of primary adnexal masses by 3 T MRI: categorization with conventional MR imaging and diffusion-weighted imaging. J Ovarian Res. 2012;5:33.

22. Min Q, Shao K, Zhai L, Liu W, Zhu C, Yuan L, et al. Differential diagnosis of benign and malignant breast masses using diffusion-weighted magnetic resonance imaging. World J Surg Oncol. 2015;13:32.

23. Caraiani C, Chiorean L, Fenesan DI, Lebovici A, Feier D, Gersak M, et al. Diffusion weighted magnetic resonance imaging for the classification of focal liver lesions as benign or malignant. J Gastrointestin Liver Dis. 2015;24:309-17.

24. Göya C, Hamidi C, Bozkurt Y, Yavuz A, Kuday S, Gümüş H, et al. The role of apparent diffusion coefficient quantification in differentiating benign and malignant renal masses by 3 Tesla magnetic resonance imaging. Balkan Med J. 2015;32:273-8.

25. Oh SN, Rha SE, Byun JY, Lee YJ, Jung SE, Jung CK, et al. MRI features of ovarian fibromas: emphasis on their relationship to the ovary. Clin Radiol. 2008:63:529-35.

26. McGee J, Fleming NA, Senterman M, Black AY. Virilizing luteinized thecoma of the ovary in a 15-year-old female: a case report. J Pediatr Adolesc Gynecol. 2009;22:107-10.

27. Roth $L M$, Gaba $A R$, Linden MD, Sandusky GE. Involuting luteinized thecoma of the ovary. Int J Gynecol Pathol. 2014;33:23-9. 